\title{
Original
}

\section{A Preliminary Study of the Relationship between Oral and Vascular Function in the Elderly Population}

\author{
Kei Suzuki' ${ }^{1)}$ Hiroyuki Nakano ${ }^{1)}$, Nahoko Kato-Kogoe ${ }^{1)}$, Kazuya Inoue $^{1)}$, Michi Omori ${ }^{1)}$, Naoko Imagawa ${ }^{1)}$, Yoshifumi Suwa ${ }^{1)}$, \\ Kayoko Yamamoto ${ }^{2)}$, Kuniyasu Kamiya ${ }^{3)}$, Satoyo Ikehara $^{3)}$, Junko Tamaki ${ }^{3)}$, Masaaki Hoshiga ${ }^{4)}$ and Takaaki Ueno ${ }^{1)}$ \\ 1) Department of Oral Surgery, Osaka Medical College, Osaka, Japan \\ 2) Department of Oral Biological \& Medical Sciences, Faculty of Dentistry, The University of British Columbia, Vancouver, Canada \\ 3) Department of Hygiene and Public Health, Osaka Medical College, Osaka, Japan \\ 4) Department of Cardiology, Osaka Medical College, Osaka, Japan \\ (Accepted for publication, April 12, 2020)
}

\begin{abstract}
The goal of hard tissue reconstruction in the oral and maxillofacial field is suitable oral function in elderly patients. Recently, some studies have reported an association between cardiovascular diseases and oral function. However, these have not been supported by extensive evidence and further collection of data is needed. This study included 108 residents of Takatsuki City who were 65 years of age or older. Oral environment and function (number of teeth, maximum occlusal force, gummy score, and chewing time), vascular functions (brachial-ankle pulse wave velocity, baPWV; and ankle brachial pressure index, $\mathrm{ABI}$ ) were measured. Negative correlations were observed between the number of teeth and baPWV, maximum occlusal force and baPWV, and gummy score and baPWV. A negative correlation was observed between chewing time and rtABI. Multiple linear regression analysis showed that maximum occlusal force had significant negative associations with baPWV (standardized partial regression coefficient $(\beta)=-0.251, p<0.05$ ) after controlling for age. The present study suggested some degree of association between oral function and cardiovascular diseases. A decline in masticatory efficiency may limit the type of foods that can be ingested, leading to changes in dietary habits and associated imbalances in nutritional intake. Furthermore, progression of periodontal disease caused by reduced salivary secretion may influence vascular sclerosis, thus supporting the development of cardiovascular diseases. Further research is needed to determine the causal relationship.
\end{abstract}

Key words: Gummy score, Masticatory efficiency, Maximum biting force, Number of existing permanent teeth, Vascular function

\section{Introduction}

Cardiovascular disease and cerebrovascular disease were the second and fourth leading causes of death in Japan in $2017^{1)}$. Until 2010, cerebrovascular disease was one of the three leading causes of death in Japan. While pneumonia has since taken its place, cerebrovascular disease remains the third highest cause of death among people between the ages of 60 and 79 years. Many patients develop motor impairment and eating/swallowing disorder as a sequela, which comprises the primary factor associated with need for long-term care. The risk of death by cardiovascular disease tends to increase with age. Thus, cardiovascular disease cannot be ignored when addressing public health issues of Japanese people. Therefore, as Japan has become a super-aging society, identifying the causes and methods to prevent onset of cardiovascular diseases are important research topics ${ }^{2}$. Furthermore, the goal of hard tissue reconstruction in the oral cavity region is recovery of proper oral function. Some studies have reported an association of cardiovascular disease with maintenance of oral and masticatory function ${ }^{3)}$. However, such an association is not greatly supported by the available evidence; thus, fur-

Correspondence to: Dr. Kei Suzuki, Division of Medicine for Function and Morphology of Sensor Organ, Dentistry and Oral Surgery, Osaka Medical College, 2-7 Daigaku-machi. Takatsuki City, Osaka 569-8686 Japan; Tel: +8172-683-1221; Fax: +81-72-684-1422; E-mail: ora096@osaka-med.ac.jp ther data collection and analysis is needed. This study measured oral function and cardiovascular disease characteristics in individuals over the age of 65 years, in order to assess the association between oral function and vascular sclerosis.

\section{Participants}

\section{Materials and Methods}

Subjects of the present study were 53 men and 55 women aged 65 years or older living in Takatsuki City (108 subjects). They underwent the following examination in November 2017.

\section{Examination items}

All participants completed individual interviews, followed by oral examination by dentists. The following items were assessed.

\section{Oral function}

- Number of teeth

-Maximum occlusal force: Maximum occlusal force was measured once for each side using a simple occlusal force measuring device (Occlusal Force Meter GM10; Nagano Keiki Co., Ltd., Tokyo, Japan) to determine the maximum value for each subject.

- Masticatory performance (Gummy score): Gummy jelly score was 
used to assess mastication ability. To obtain gummy jelly scores, each subject chewed one test gummy jelly (UHA Mikakuto Co., Ltd., Osaka, Japan) as the chewing sample 30 times, then spit the broken pieces onto a gauze cloth. The pieces were transferred to a transparent petri dish and used as samples for visual inspection by two evaluators who visually compared the sizes of the pieces in the same sample on a scale of 1 to 10 , in order to obtain a gummy score $^{4,5)}$. If the scores of the two evaluators differed, the higher score was used.

-Chewing time: Mastication time for 30 chews was measured.

\section{Vascular function}

Vascular function was assessed using brachial-ankle pulse wave velocity (baPWV) and ankle brachial pressure index (ABI).

-Brachial-ankle pulse wave velocity (baPWV) (EndoPAT; Nihon Kohden Corporation, Tokyo, Japan): The cuffs were wrapped around both sides of the upper arms and ankles and pressure was applied. After recording the volume pulse waveforms, baPWV was calculated by first determining the time difference between the onset of the rising pulse waveform of the brachial and ankle waveforms, then dividing it by the difference in distance between the heart and the measurement location. The max value between the right and left baPWV was used as the representative value.

-Ankle brachial pressure index (ABI): The cuffs were wrapped around both ankles and the systolic blood pressures of both dorsalis pedis and posterior tibial arteries on both lower limbs were measured using the Doppler method. ABI was calculated by dividing the higher blood pressure by the brachial systolic blood pressure (either the right or left).

\section{Correlations between variables}

The correlation coefficients for age, number of teeth, maximum occlusal force, masticatory performance, chewing time, baPWV, and ABI were calculated.

\section{Ethical considerations}

This study was approved by the Ethics Committee of Osaka Medical College (approval \#2309). All participants provided informed consent to undergo oral examinations and functional assessments.

\section{Statistical analysis}

We conducted statistical analysis using Spearman's rank-order correlative coefficient and multiple linear regression analyses to evaluate the associations between indicators of oral function and vascular function. The level of significance was set as $\mathrm{p}<0.05$.

\section{Results}

A total of 108 elderly adults participated in this study; of these, 53 were men and 55 were women, with a mean age of $75.5 \pm 4.8$ years. Summary of oral and vascular conditions of these participants, such as the number of teeth, maximum occlusal force, masticatory performance, chewing time, baPWV, and ABI are shown in Table 1.

To evaluate the associations between indicators of oral function and vascular function, we conducted statistical analysis. Negative correlations were observed between number of teeth and baPWV, maximum occlusal force and baPWV, masticatory performance and baPWV (Spearman's rank correlation coefficient; $r_{s}=-0.264,-0.226$, and -0.206 , respectively: $\mathrm{p}<0.05$ ) (Fig 1, 2, and 3). In addition, A negative correlation was observed between chewing time and rABI (Spearman's rank
Table 1. Summary of oral and vascular conditions

\begin{tabular}{ll}
\hline Examination item & Median (min-max) \\
\hline Number of teeth (number) & $23.0(0-28)$ \\
Maximum occlusal force $(\mathrm{N})$ & $245.5(11-1090)$ \\
Masticatory performance (Gummy jelly score) & $5(0-9)$ \\
Chewing time (s) & $25.0(17-69)$ \\
Brachial-ankle pulse wave velocity (cm/sec) & $1692.0(1107.0-2817.0)$ \\
Right Ankle brachial pressure index & $1.14(1.04-1.29)$ \\
Left Ankle brachial pressure index & $1.13(0.96-1.30)$ \\
\hline
\end{tabular}

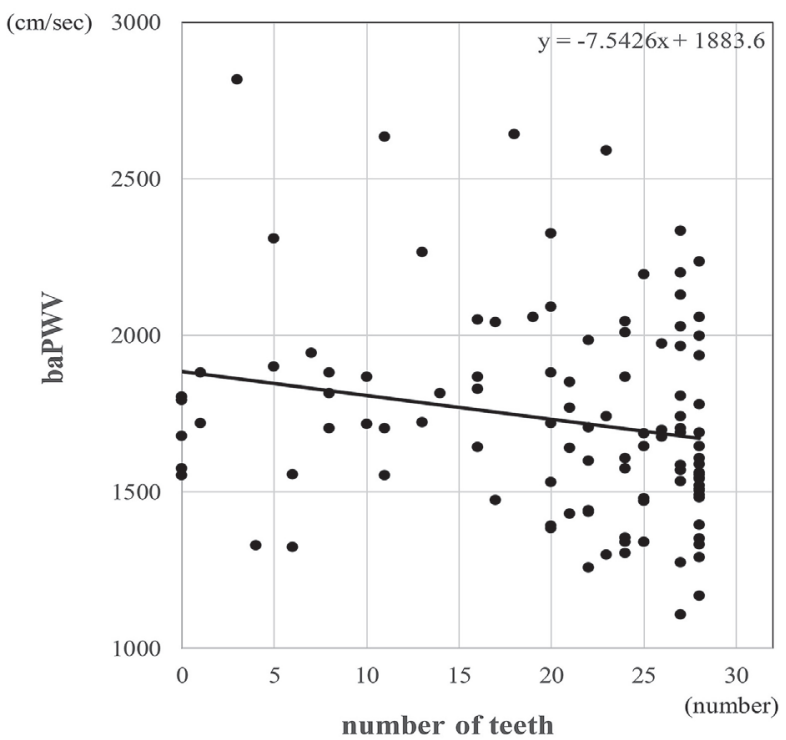

Figure 1. Correlation between number of teeth and brachial-ankle pulse wave velocity (baPWV). There is a negative correlation between number of teeth and baPWV.

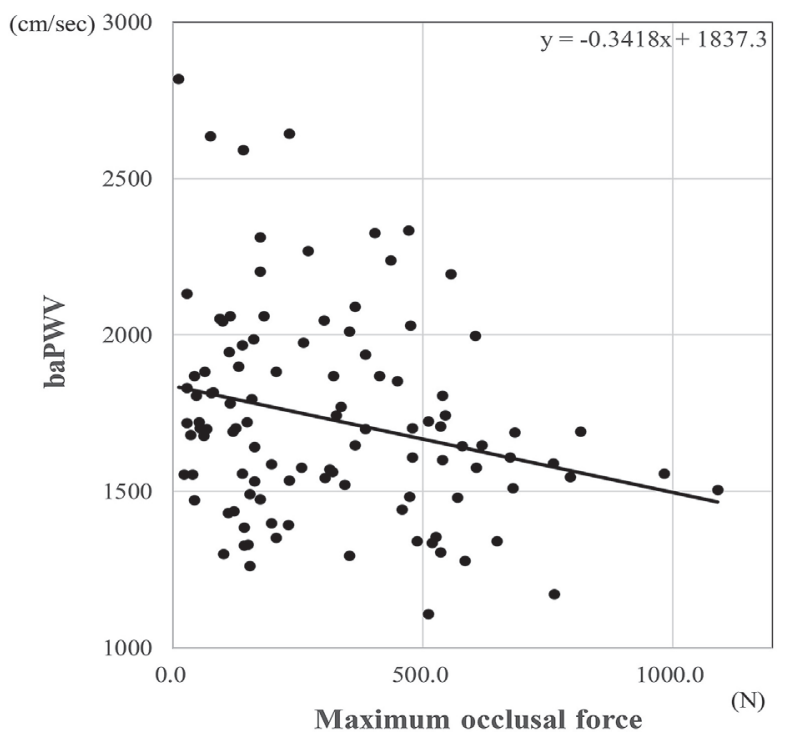

Figure 2. Correlation between maximum occlusal force and. brachial-ankle pulse wave velocity (baPWV). There is a negative correlation between maximum occlusal force and baPWV. 
Kei Suzuki et al.: Oral and Vascular Function in the Elderly

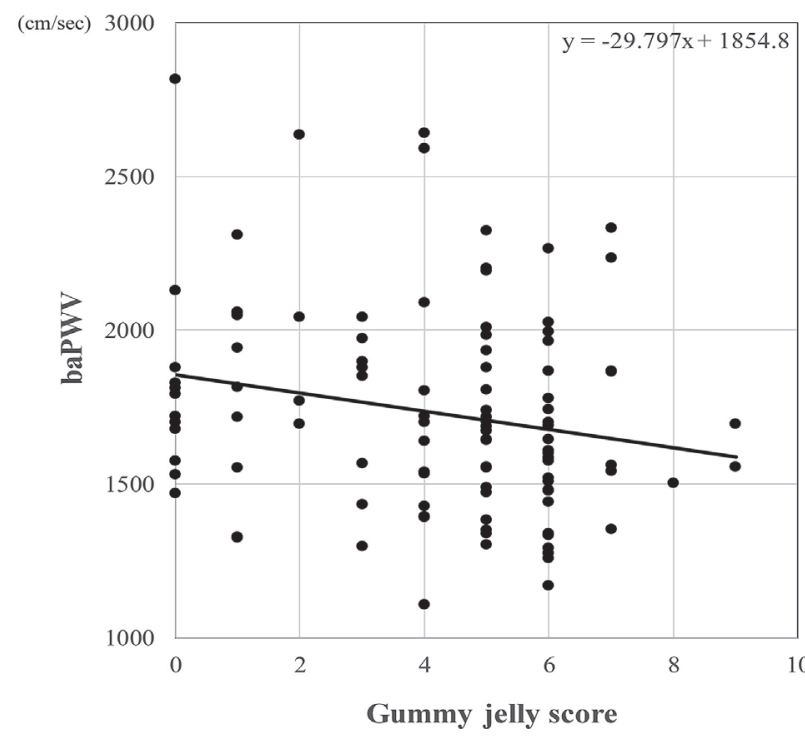

Figure 3. Correlation between gummy jelly score and brachial-ankle pulse wave velocity (baPWV). There is a negative correlation between gummy jelly score and baPWV.

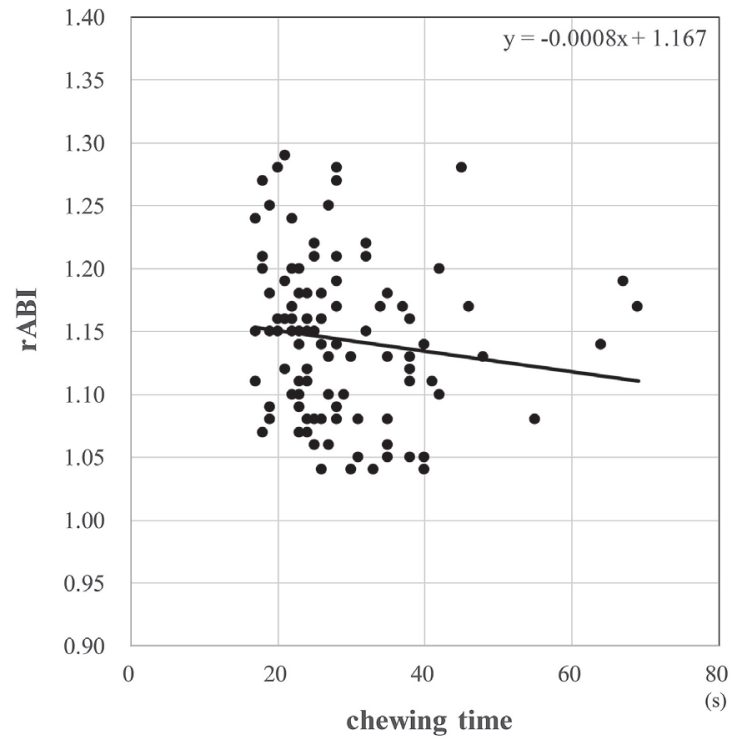

Figure 4. Correlation between chewing time and right ankle brachial pressure index $(\mathrm{ABI})$. There is a negative correlation between chewing time and $\mathrm{rABI}$.

correlation coefficient; $r_{s}=-0.223: p<0.05$ ) (Fig 4), whereas significant correlation was not observed between chewing time and $1 \mathrm{ABI}$.

Moreover, we performed multiple linear regression analysis. The results showed that maximum occlusal force had significant negative associations with baPWV (standardized partial regression coefficient $(\beta)=$ $-0.251, \mathrm{p}<0.05)$ after controlling for age.

\section{Discussion}

Japan is experiencing an unprecedented super-aging of its society. The aging rate (percentage of the population that is 65 years and older) will be approximately $30 \%$ in 2025 and will reach approximately $40 \%$ by 2060 . The arrival of a super-aging society is likely linked with increased future risks of cardiovascular diseases. From this perspective, the management of arteriosclerotic risk factors in the elderly and the establishment of a means of preventing these cardiovascular diseases are important issues ${ }^{6}$.

Among a variety of cardiovascular diseases, myocardial infarction and angina pectoris, which results from ischemia of the myocardium, are collectively known as ischemic heart diseases. An increase in the incidence of metabolic diseases, such as diabetes and hypertension, which is attributed to the growing westernization of the diet and lifestyles, has led to increased prevalence of ischemic heart diseases.

Arteriosclerosis, which is the cause of ischemic heart diseases, is believed to result from chronic vascular inflammation. In recent years, pathological conditions, such as diabetes and hypertension, known to be involved in arteriosclerosis, have also been associated with chronic tissue inflammation. Notably, these diseases have been attributed to systemic inflammation. The involvement of tissues adjacent to arteriosclerotic lesion sites has also gained attention, particularly with regard to the effects of arteriosclerosis-promoting factors at lesion sites; these include bone marrow cells, microvessels in the adventitia, perivascular adipose tissue, and oral microbial flora ${ }^{7}$.

While there is a high prevalence and associated high mortality rate of ischemic heart disease among patients with periodontal disease and those who have lost a large number of teeth, there is insufficient evidence regarding the association between the incidence of periodontal disease and the progression of ischemic heart disease ${ }^{8)}$. However, the present study has suggested an association between oral function and arteriosclerosis. A decline in masticatory efficiency may limit the types of foods that can be ingested, leading to changes in dietary habits and associated imbalances in nutritional intake. Furthermore, progression of periodontal disease caused by reduced salivary secretion may influence vascular sclerosis, thereby leading to cardiovascular diseases ${ }^{9}$. Further research is necessary to determine whether there is a causal relationship.

\section{Acknowledgments}

This research was partially supported by Private University Research Branding Project 2017 and Japan Society for the Promotion of Science KAKENHI Grant Number JP18K17213, JP19K06873.

\section{Conflict of Interests}

The authors have declared that no COI exists.

\section{References}

1. Cabinet Office, Japan. Koureisyakaihakusyo: 2-7, 2017

2. Holmstrup P, Poulsen AH and Andersen L. Oral infections and systemic diseases. Dent Clin North Am 47: 575-598, 2003

3. Bahekar AA, Singh S, Saha S, Molnar J and Arora R. The prevalence and incidence of coronary heart disease is significantly increased in periodontitis. a meta-analysis. Am Heart J 154: 830-837, 2007

4. Nokubi T, Yoshimuta Y, Nokubi F, Yasui S, Kusunoki C, Ono T, Maeda Y and Yokota K. Validity and reliability of a visual scoring method for masticatory ability using test gummy jelly. Gerodontology 30: 76-82, 2012

5. Nakajima Y, Ueno T, Kato-Kogoe N, Yamamoto K, Fukui N, Suwa Y, Kimura Y, Ito Y, Takahashi Y, Kasuya S, Kanou M, Terai H, Tano T, Ariyoshi Y. Study on suitability of grafted bone following mandibular reconstruction evaluated according to masticatory performance scores using half-potion gummy jelly. J Hard Tissue Biol 25: 427430, 2016

6. Noack B, Genco RJ and Trevisan M. Periodontal infections contrib- 
ute to elevated systemic C-reactive protein level. J Periodontol 72: 1221-1227, 2001

7. Ridker PM, Cushman M and Stampfer MJ. Inflammation, aspirin, and the risk of cardiovascular disease in apparently healthy men. N Engl J Med 336: 973-979, 1997

8. Taniguchi A, Nishimura F and Murayama Y. Porphyromonas gingivalis infection is associated with carotid atherosclerosis in nonobese Japanese type 2 diabetic patients. Metabolism 52: 142-145,
2003

9. Inomata C, Ikebe K, Kagawa R, Okubo H, Sasaki S, Okada T, Takeshita H, Tada S, Matsuda K, Kurushima Y, Kitamura M, Murakami S, Gondo Y, Kamide K, Masui Y, Takahashi R, Arai Y and Maeda Y. Significance of occlusal force for dietary fibre and vitamin intakes in independently living 70-year-old Japanese. J Dent 42: 556-564, 2014 